\title{
Das Gedächtnis ist am Zug
}

\author{
Adrian Siegel ist Präsident des Schweizer Schachbundes. Als Neurologe untersucht er die Auswirkungen \\ des Brettspiels auf das Gehirn auch im MRI - und empfiehlt es seinen Patienten bisweilen als Therapie.
}

\section{Adrian Ritter}

Freier Journalist

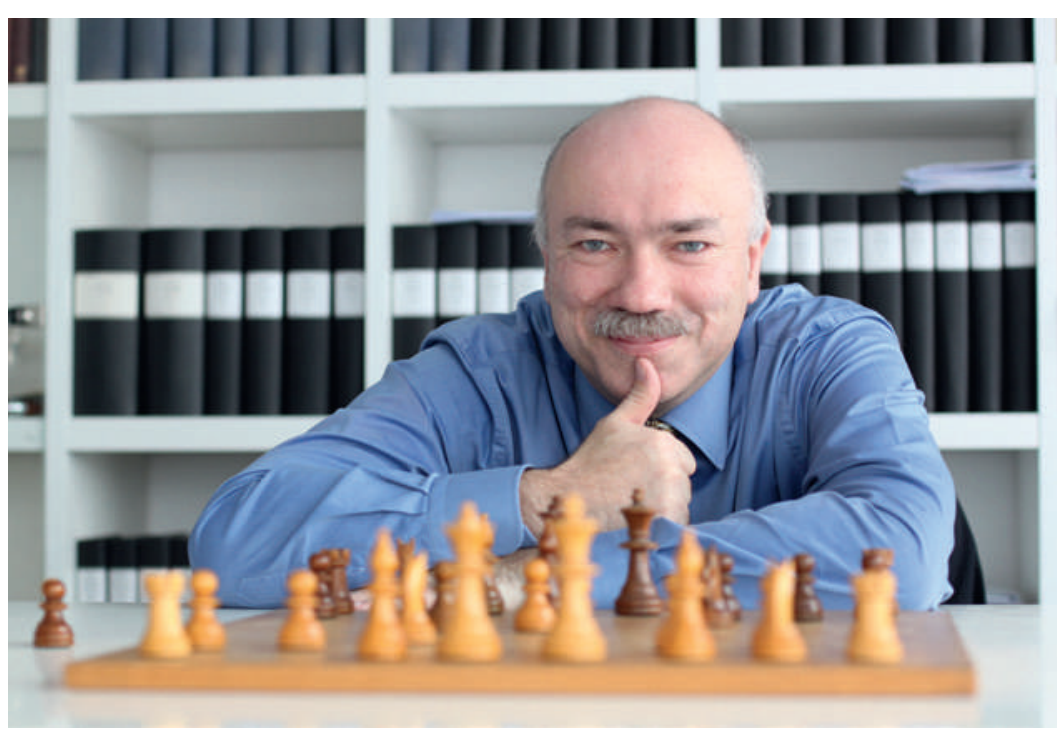

Der Neurologe Adrian Siegel: «Schach ermöglicht Momente der intellektuellen Ruhe und Entspannung. Ich möchte diese Erfahrung nicht missen.»

Es war ein verlockender Deal: Eine Briefmarke für jede gewonnene Partie. So konnte ein Freund der Familie den damals 6-jährigen Adrian Siegel für das Schachspiel interessieren. Es sollte eine nachhaltige Begeisterung und Schach bald wichtiger werden als die Briefmarkensammlung. Rasch machte der junge Spieler Fortschritte.

Heute ist Adrian Siegel (51) Vorstandsmitglied im Weltschachverband (FIDE) und noch bis Juni 2015 Präsident des Schweizer Schachbundes. Viel Zeit für das Schachspielen im Freundeskreis und in seinem Schachclub hat er allerdings nicht. Neben seinem Engagement im Verbandswesen ist er in Thalwil als Neurologe in eigener Praxis mit den Schwerpunkten Epilepsie, Kopfschmerz und Schlaganfall tätig. So finden die Schachzüge derzeit eher im Kopf statt, wenn er etwa vor dem Einschlafen eine Spieleröffnung mental durchgeht. «Es gab Zeiten, da spielte ich rund 100 Turnierspiele pro Jahr», sagt er. Um weitere Fortschritte zu machen, hatte er sich 2008 gar einen ehemaligen JuniorenWeltmeister als Trainer genommen. In jener Zeit des intensiven Übens erlebte er, wie Schach sein Gedächtnis verbesserte. Beim Zoobesuch konnte er sich plötzlich an die französischen Namen der Tiere erin- nern - Wörter, die er seit der Gymnasialzeit nie mehr verwendet hatte. Dass sich das Gedächtnis auch ausserhalb des trainierten Feldes verbessert, solche "Transformationseffekte» sind wissenschaftlich umstritten. «Ich habe sie erlebt», schmunzelt Siegel.

\section{Schachspieler in der Röhre}

Er hat noch mehr gesehen, denn das Schachspiel hat ihn auch als Neurologe je länger, je mehr fasziniert: Was genau geschieht im Gehirn? Lässt sich der Trainingseffekt in der Hirnstruktur nachweisen? Siegel wollte es genau wissen und lancierte ein Forschungsprojekt an der Universität Zürich, wo er auch Titularprofessor ist. Die Forschungsgruppe verglich je 20 Profi- und Amateurspieler mittels Magnetresonanztomographie und stellte fest: Im Gehirn der Profis sind Hippocampus und assoziativer Cortex vergrössert. Schachspiel schult das Gedächtnis und die Fähigkeit, Zusammenhänge herzustellen - und scheint dabei wirksamer zu sein als etwa jassen oder Sudoku lösen. Ein hoher Intelligenzquotient sei für das Schachspiel weder nötig, noch lasse er sich durch das Spielen steigern, sagt Siegel: «Was es braucht, sind Gedächtnis, Konzentration, Aufmerksamkeit, Vorstellungsvermögen und Selbstdisziplin. Und all dies lässt sich im Spiel auch trainieren.» Zudem ist der Neurologe mit dem früheren US-Präsidenten Benjamin Franklin einig, der 1786 in einem Essay das Schachspiel als Charakterschulung pries. Man lerne, sich an Regeln zu halten, zu verlieren und Respekt vor dem Gegner zu zeigen.

Weil sich all dies beim Schach auf spielerische Weise ergebe, sähe Adrian Siegel das Brettspiel am liebsten als Schulfach. Als Neurologe hat er Schach auch schon therapeutisch empfohlen: «Es gibt Hinweise, dass sich bei Demenz der Verlust der Gedächtnisleistung damit verlangsamen lässt.»

In einem weiteren Forschungsprojekt möchte Siegel untersuchen, ob bei Frauen und Männern unterschiedliche Hirnareale aktiviert werden beim Schach: Spielen Frauen intuitiver? Setzen sie eher als Männer beide Hirnhälften ein? Dass unter den weltweit 50 besten Spielern keine Frauen zu finden sind, habe vor allem 
mit sozialen Faktoren zu tun, vermutet Siegel. Bisweilen chauvinistische männliche Schachspieler schreckten Frauen früher ab und es fehle bis heute an einer konsequenten Förderung weiblicher Talente.

\section{Mit stechendem Blick}

Wenn beim Schachspiel das Gehirn eine so grosse Rolle spielt, ist Adrian Siegel mit seinem neurologischen Wissen dann am Brett im Vorteil? Er lacht. «Nein, ich glaube nicht. Allenfalls lasse ich mich von psychologischen Tricks weniger beeindrucken.» Gewisse Spieler versuchen, den Gegner durch Anstarren zu irritieren. Oder sie machen ihn schlicht mit ihren Macken unabsichtlich nervös - mit minutenlangem Rühren im Kaffee oder Vor-sich-hin-Pfeifen etwa. Siegel weist dezent darauf hin, wenn ihn etwas stört. Als Teenager hatte er es selber versucht mit dem stechenden Blick den russischen Schachgrossmeister Anatoli Karpow nachahmend. "Ich merkte aber bald, dass mich dieses Verhalten selber mehr ablenkt als den Gegner und gab es auf», lacht er rückblickend.

\section{Im Gehirn der Profis sind Hippocampus und assoziativer Cortex vergrössert.}

Wenn Macken eher störend wirken, so sind doch Rituale beim Schachspiel wichtig, sagt Siegel. Er achtet vor allem auf eine angepasste Ernährung, denn eine durchschnittlich vierstündige Turnierpartie zehrt an den Reserven: Unterzuckerung droht. «Die Fehlerquote nimmt stetig zu, je länger das Spiel dauert. Nach mehreren Stunden sieht man das Brett vor sich bisweilen leicht verschwommen.» Schokoriegel und Eistee sollen ihm helfen, konzentriert zu bleiben. Es sind weniger medizinische Erkenntnisse, die er sich dabei zu Herzen nimmt als die Tipps der Schachweltmeister: Nach 15 Spielminuten ein zuckerhaltiges Getränk oder etwa eine Banane zu sich nehmen. Zwischendurch auf die Toilette gehen, um dem Gehirn eine Verschnaufpause zu gönnen. Die Hände zwischen Stuhl und Oberschenkel klemmen, um keine voreiligen Züge zu tätigen, wenn die Zeit knapp wird.

\section{Das Gedächtnis dopen}

Sich im Schachspiel dopen zu wollen, würde wohl wenig bringen, ist Siegel überzeugt. Ritalin könnte allenfalls für eine erhöhte Konzentration sorgen und ist deshalb bei Turnieren verboten. Wer aus medizinischen Gründen eine Ausnahme braucht, muss beim Verbandsarzt des Schachbundes vorstellig werden. «Bei anderen Medikamenten lässt die konzentrations- fördernde Wirkung bereits nach ein bis zwei Stunden nach. Danach plagen einen Nebenwirkungen, die eher für einen wirren Kopf sorgen», so Siegel.

In seiner früheren Funktion als Mitglied der Ärztekommission des Weltschachverbandes hatte er nie Dopingfälle erlebt. Dass Betablocker bis kürzlich bei Weltmeisterschaften verboten waren, betrachtete er schon immer als unsinnige Regel, die höchstens Probleme für Blutdruckpatienten mit sich brachte. Die Welt-Anti-Doping-Agentur (WADA) hat diese Vorgabe jetzt gestrichen. Falls Schach aber zur Olympischen Disziplin wird, wie das der Weltschachverband anstrebt, wird die Liste der verbotenen Medikamente und Stoffe wieder länger werden.

\section{Ärzte mit Zeitmangel}

Adrian Siegel war nie versucht, seinen Leistungen im Schachspiel mit Substanzen nachzuhelfen. «Für mich ist Schach in erster Linie ein Spiel. Natürlich macht es Freude, wenn es nach Stunden gelingt, einen minimalen Vorteil in ein Schachmatt zu verwandeln. Aber im Vordergrund steht die Geselligkeit im Club und die intellektuelle Herausforderung."

Das Spiel vermag alle sozialen und intellektuellen Schichten zu begeistern, stellt er im Schachbund fest. Leicht übervertreten seien Akademiker - vor allem solche aus den Bereichen Mathematik und Informatik. Und Ärztinnen und Ärzte? "Ich begegne leider nicht vielen. Es fehlt ihnen vermutlich an der Zeit oder sie haben schon genügend andere gesellige Anlässe», so Siegel.

Aber wer weiss, vielleicht werden einige Ärzte, die bisher nur im privaten Kreis spielten, diesen Sommer plötzlich in Biel anzutreffen sein. Dort findet am 25. Juli im Rahmen des Internationalen Schachfestivals erstmals ein eigenes Turnier für Ärzte statt. In Deutschland sind Mediziner schon seit 22 Jahren die einzige Berufsgruppe, die ein eigenes Turnier kennt. Siegel würde sich über neue Kolleginnen und Kollegen aus der Ärzteschaft freuen: «Schach ermöglicht Momente der intellektuellen Ruhe und Entspannung. Ich möchte diese Erfahrung nicht missen.»

\section{Ärzteturnier am Internationalen Schachfestival}

Vom 18. bis 31. Juli 2015 findet in Biel das 48. Internationale Schachfestival statt. Zum ersten Mal wird dabei für Ärztinnen und Ärzte am 25. Juli ein separates Turnier angeboten. In einem "Simultan» können Ärztinnen und Ärzte zudem an mehreren Brettern gleichzeitig gegen einen Grossmeister spielen. www.bielchessfestival.ch 